\title{
Histopathological Findings of Intracranial Thrombi in Nonbacterial Thrombotic Endocarditis
}

\author{
Kyoungsub Kim, ${ }^{\mathrm{a}, \mathrm{b}}$ Jayoung Kim, ${ }^{\mathrm{c}}$ Seong Hwan Ahn, ${ }^{\mathrm{d}}$ Woo Seok Ha, ${ }^{\mathrm{a}} \mathrm{Yu}$ Jin Koo, ${ }^{\mathrm{a}}$ \\ Dong Joon Kim, ${ }^{\mathrm{e}}$ Hyo Suk Nam, ${ }^{\mathrm{a}} \mathrm{Ji} \mathrm{Hoe} \mathrm{Heo}^{\mathrm{a}}$ \\ aDepartment of Neurology, Yonsei University College of Medicine, Seoul, Korea \\ bDepartment of Neurology, Mediplex Sejong Hospital, Incheon, Korea \\ 'Severance Integrative Research Institute for Cerebral \& Cardiovascular Disease, Yonsei University Health System, Seoul, Korea \\ dDepartment of Neurology, Chosun University School of Medicine, Gwangju, Korea \\ 'Department of Radiology, Yonsei University College of Medicine, Seoul, Korea
}

\section{Dear Sir:}

Nonbacterial thrombotic endocarditis (NBTE), a form of endocarditis in which noninfectious vegetations are deposited on cardiac valves, is a cause of stroke. NBTE is frequently related to malignancies and hypercoagulable conditions. ${ }^{1,2}$ Since previous pathological investigations of NBTE have been based on postmortem studies of cardiac valve vegetations, ${ }^{1,3}$ the histological features of intracranial arterial thrombi in NBTE remain unknown. We present the histological features of thrombi, which were retrieved by using a stent retriever, in a patient with stroke and NBTE.

A 55-year-old man was admitted for multiple cerebral and cerebellar infarctions (Supplementary Figure 1A). He had no relevant medical history. The findings of 12-lead electrocardiography and continuous electrocardiographic monitoring, during his stay in the stroke unit, were normal. Blood chemistry, coagulation, and autoimmunity test results were within the normal ranges, except for an elevated level of D-dimer $(7,251 \mathrm{mg} / \mathrm{mL}$ ) (Supplementary Figure 2). Transesophageal echocardiography showed echogenic masses on the anteromedial leaflet of mitral valve and the non-coronary cusp of aortic valve, which were compatible with NBTE (Figure 1A). Positron emission tomography-computed tomography (CT) and abdominopelvic CT showed multiple metastatic lymph nodes with F-18 fluorodeoxyglucose uptake in the portacaval, aortocaval, and para-aortic areas. Laparoscopic lymph node biopsy showed moderately differentiated metastatic adenocarcinoma. The pa- tient was diagnosed with primary unknown cancer. He was treated with intravenous heparin and advised to take rivaroxaban following discharge (15 mg bid) as he had concomitant pulmonary embolism. He developed small multiple scattered infarctions in the bilateral cerebellar and cerebral hemispheres (Supplementary Figure 1B), 4 days after discharge. Subcutaneous enoxaparin ( $60 \mathrm{mg}$ bid) was administered. However, 6 days later, he developed sudden weakness on his left side. CT angiography showed occlusion of the right proximal middle cerebral artery. Endovascular thrombectomy was performed with Solitaire (ev3, Irvine, CA, USA). The artery was successfully recanalized after five passes, but developed a large infarction (Supplementary Figure 1C). The retrieved pieces of thrombi were whitish and rubbery (Figure 1B). We performed hematoxylin-eosin and immunohistochemical staining for platelets, fibrin/fibrinogen, red blood cells, and neutrophils. Details of the staining methods are described in the Details of the staining methods in the supplementary data (Supplementary Method 1). Histologic examinations demonstrated that the entire thrombus area comprised platelets interwoven with thin fibrin strands. The fibrins also lined the edge of the thrombus. There were few neutrophils and red blood cells (Figure $1 C-G$ ).

The thrombi in this case were rich in platelets. To our knowledge, this is the first report demonstrating the histopathological features of intracranial thrombi in NBTE. Previous pathological examination of cardiac valve vegetations in NBTE showed platelet thrombi interwoven with fibrin strands, without leukocytes, ${ }^{1,3}$ The histological features of thrombi retrieved 

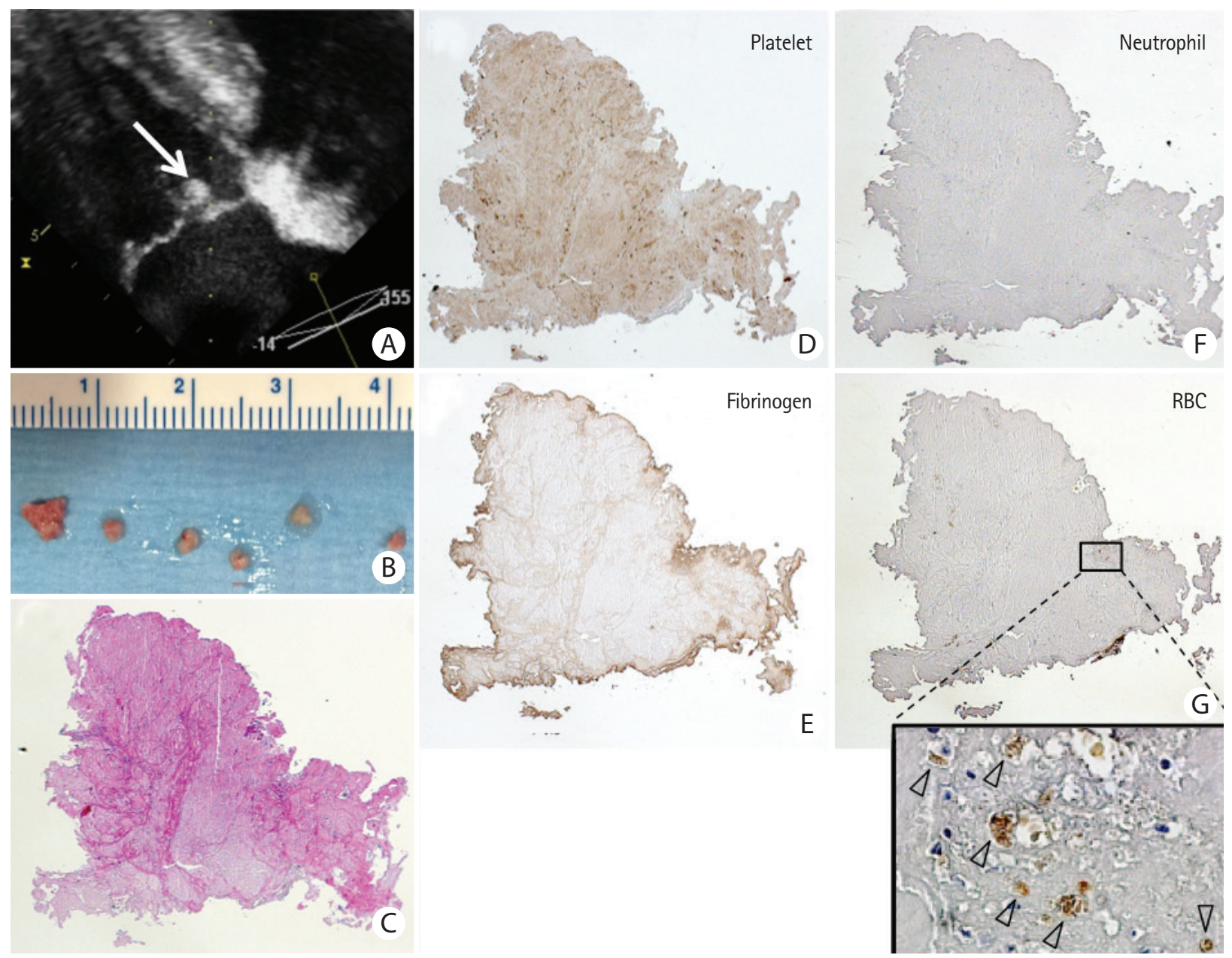

Figure 1. (A) Transesophageal echocardiogram showing a 0.6×0.5-cm echogenic mass on the anteromedial leaflet of the mitral valve (arrow). (B) Retrieved thrombi. (C) Hematoxylin-eosin staining of the thrombus. (D-G) Immunohistochemical analyses with antibodies against CD42b, fibrinogen, myeloperoxidase, and glycophorin $\mathrm{A}$, to visualize platelets, fibrin/fibrinogen, neutrophils, and red blood cells (RBCs), respectively. Positive signals were indicated by the development of a brown color. Abundant platelets were seen in the entire thrombus (D); fibrinogen/fibrin appeared at the edge or as thin strands within the thrombus (E); neutrophils were hardly visible (F); and RBCs were present (arrowheads) (G).

in the current case were similar to those of the cardiac valve vegetations in previous reports of NBTE, which suggested that the thrombi were embolized from the vegetation in the mitral or aortic valve. Vegetations in NBTE were observed on leaflets of the mitral or aortic valves where a high-flow condition exists. ${ }^{2}$ Red thrombi are known to be produced in low-flow conditions, whereas white thrombi are produced in high-flow conditions. ${ }^{4}$ Thus, high-shear stress might have contributed to the formation of platelet-rich thrombi in NBTE.

The patient described in this report developed stroke three times within 1 month. Furthermore, the last two attacks recurred during anticoagulation therapy. In the past, intravenous or subcutaneous heparin has been empirically recommended for stroke prevention in NBTE. ${ }^{5}$ Anticoagulants are usually effective in preventing the formation of slow-flow-related red thrombi. In contrast, antiplatelet agents are effective against high-flow-related white thrombi. Experiences similar to the present case are limited. However, the histological features of platelet-rich thrombi, occurrence of vegetation at high-flow locations, and ineffective anticoagulation for stroke prevention highlighted whether anticoagulation should be the treatment of choice for stroke prevention in patients with NBTE. Antiplatelet agents may be an alternative treatment for stroke prevention, at least in cancer-associated NBTE.

\section{Supplementary materials}

Supplementary materials related to this article can be found online at https://doi.org/10.5853/jos.2017.00696. 


\section{References}

1. Biller J, Challa VR, Toole JF, Howard VJ. Nonbacterial thrombotic endocarditis. A neurologic perspective of clinicopathologic correlations of 99 patients. Arch Neurol 1982;39:9598.

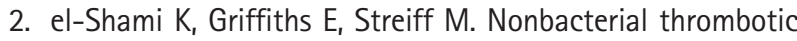
endocarditis in cancer patients: pathogenesis, diagnosis, and treatment. Oncologist 2007;12:518-523.

3. Rogers LR, Cho ES, Kempin S, Posner JB. Cerebral infarction from non-bacterial thrombotic endocarditis. Clinical and pathological study including the effects of anticoagulation. Am J Med 1987;83:746-756.

4. Lowe GD. Virchow's triad revisited: abnormal flow. Pathophysiol Haemost Thromb 2003-2004;33:455-457.

5. Salem DN, Stein PD, Al-Ahmad A, Bussey HI, Horstkotte D,

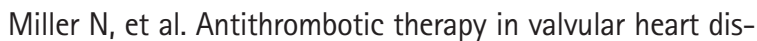
ease--native and prosthetic: the seventh ACCP conference on antithrombotic and thrombolytic therapy. Chest 2004;126(3 Suppl):457S-482S.

Correspondence: Ji Hoe Heo

Department of Neurology, Yonsei University College of Medicine, 50-1

Yonsei-ro, Seodaemoon-gu, Seoul 03722, Korea

Tel: +82-2-2228-1605

Fax: +82-2-393-0705

E-mail:jhheo@yuhs.ac

Received: April 5, 2017

Revised: May 10, 2017

Accepted: May 15, 2017

This research was supported by a grant from the Korea Health Technology R\&D Project, through the Korea Health Industry Development Institute (KHIDI), funded by the Ministry of Health 47 \& Welfare, Republic of Korea (HI08C2149).

The authors have no financial conflicts of interest. 
Supplementary Method 1. Immunohistochemical study.

Immunohistochemical analysis was performed on paraffin tissue sections, which were cut at a thickness of $4 \mu \mathrm{m}$. IHC$\mathrm{Tek}^{\mathrm{TM}}$ epitope retrieval solution and steamer (IHC world, Woodstock, MD, USA) were used for antigen retrieval of CD42b epitopes. After cooling from the heat, sections were treated with 0.075\% glycine in phosphate-buffered saline (PBS) for $10 \mathrm{~min}$ utes, and blotto (Tris-buffered saline containing 1\% horse serum and 5\% skim milk) was added thereafter. Following the blotto incubation, the sections were incubated with the primary antibodies for 2 hours at $37^{\circ} \mathrm{C}$ (for myeloperoxidase), or overnight at $4^{\circ} \mathrm{C}$ (for CD42b, fibrinogen, and glycophorin A). The following primary antibodies were used in this study: rabbit monoclonal anti-CD42b (1:100, ab134087; Abcam, Cambridge, UK), rabbit polyclonal anti-Fibrinogen (1:200, ab34269; Abcam), rabbit monoclonal anti-Glycophorin A (1:400, ab129024; Abcam), and mouse monoclonal anti-myeloperoxidase (1:200, MAB3174; R\&D systems, Inc., Minneapolis, MN, USA). Sections with blotto lacking a primary antibody were used as a negative control. Next, sections were incubated with secondary antibodies for 30 minutes at $37^{\circ} \mathrm{C}$, followed by $0.3 \%$ hydrogen peroxide in methyl alcohol for 20 minutes. The sections were then subjected to a 2-minute tap water wash, before being treated with an avidin-biotin-horseradish peroxidase complex (Vector Laboratories Ltd., Peterborough, Cambridgeshire, UK) for 30 minutes to amplify the signals. The signals were visualized by 3,3'-diaminobenzidine solution (PBS containing 0.05\% 3,3'-diaminobenzidinetetrahydrochloride hydrate and $0.1 \%$ hydrogen peroxide). After counterstaining with hematoxylin, the slides were mounted with Permount mounting medium (Fisher Scientific, Fair lawn, NJ, USA). Sections were viewed using Axio Imager D2 microscope and Axio Vision software (Carl Zeiss Microlmaging $\mathrm{GmbH}$, Jena, Germany). 

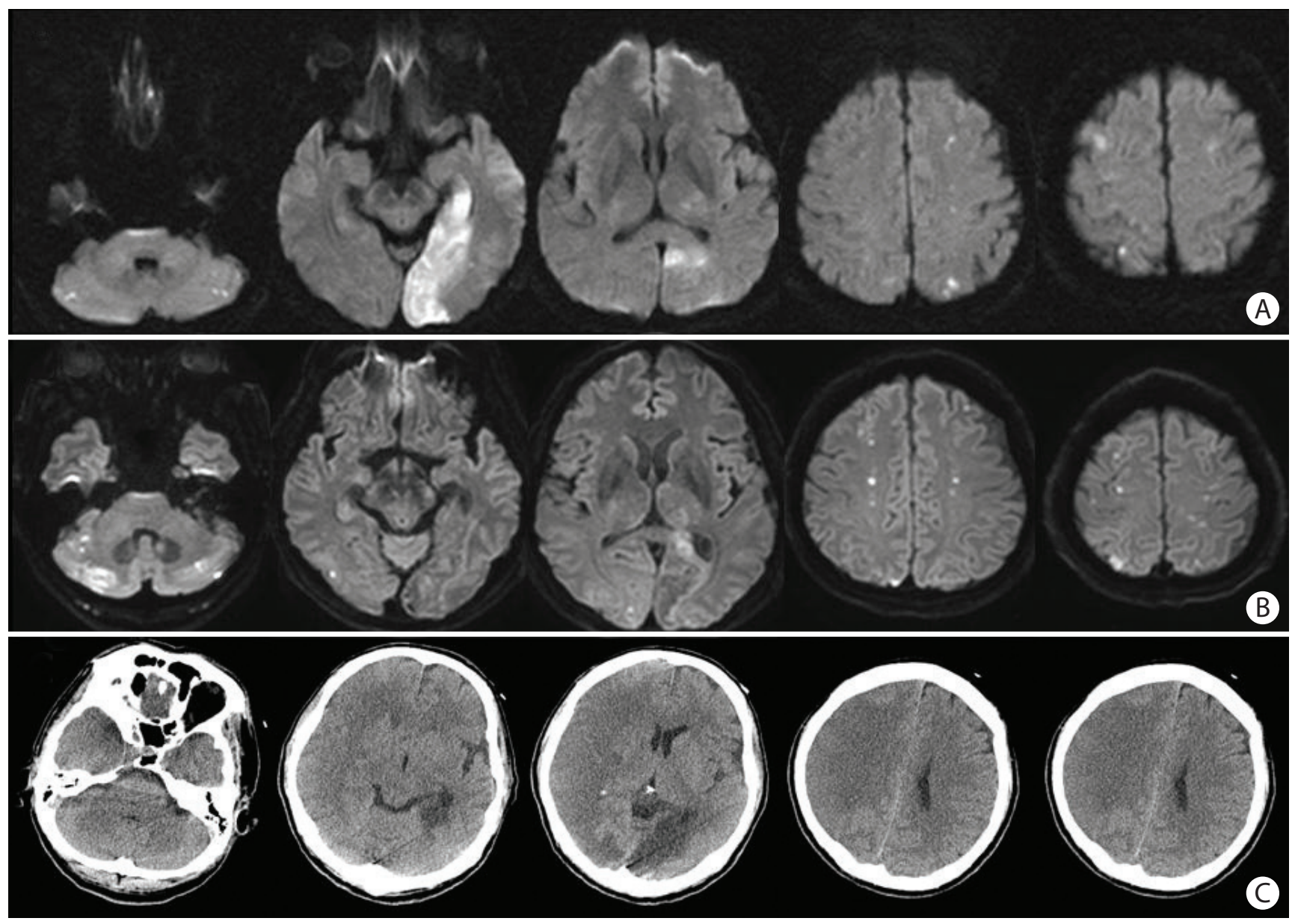

Supplementary Figure 1. Diffusion-weighted imaging (DWI) following the first attack (A) and second attack (B), and computed tomography (CT) imaging following the third attack (C). Initial DWI demonstrated a medium-sized infarction involving the left posterior cerebral artery, and many small infarctions in the bilateral cerebellar and cerebral hemispheres (A). Follow-up DWI after recurrence showed a similar pattern of infarctions (multiple small infarctions in the bilateral cerebellar and cerebral hemispheres and small-to medium sized infarction in the right cerebellum (B). The CT scan after the third attack showed a large infarction in the right middle cerebral artery territory (C). 


\begin{tabular}{lccccc} 
Event & First attack & $\begin{array}{c}\text { NBTE } \\
\text { diagnosed }\end{array}$ & & \multicolumn{2}{c}{$\begin{array}{c}\text { Second } \\
\text { attack }\end{array}$} \\
Day & 1 & 7 & 18 & 23 & 29 \\
\cline { 2 - 5 } $\begin{array}{l}\text { D-dimer } \\
(\mu \mathrm{g} / \mathrm{mL})\end{array}$ & 7251 & 5530 & 1653 & 372 \\
Treatment & $\begin{array}{c}\text { aspirin } \\
\text { clopidogrel }\end{array}$ & IV heparin & Rivaroxaban & SO enoxaparin
\end{tabular}

Supplementary Figure 2. Serial changes in the level of D-dimer and treatment modality. NBTE, nonbacterial thrombotic endocarditis; IV, intravenous; SQ, subcutaneous. 